\title{
1 Morphological transformation of soot: investigation of 2 microphysical processes during the condensation of sulfuric 3 acid and limonene ozonolysis product vapors
}

4 Xiangyu Pei ${ }^{1}$, Mattias Hallquist ${ }^{1}$, Axel C. Eriksson ${ }^{2,3}$, Joakim H. Pagels ${ }^{3}$, Neil M. Donahue ${ }^{4}$, 5 Thomas Mentel ${ }^{5}$, Birgitta Svenningsson ${ }^{2}$, William Brune ${ }^{6}$, and Ravi Kant Pathak ${ }^{1}$

${ }^{1}$ Department of Chemistry and Molecular Biology, University of Gothenburg, Gothenburg, 41296, Sweden

${ }^{2}$ Division of nuclear physics, Department of Physics, Lund University, Lund, 22100, Sweden

${ }^{3}$ Ergonomics and Aerosol Technology, Lund University, Lund, 22100, Sweden

${ }^{4}$ Center for Atmospheric Particle Studies, Carnegie Mellon University, Pittsburgh, PA, 15213, USA

${ }^{5}$ Forschungszentrum Jülich GmbH, Jülich, 52428, Germany

${ }^{6}$ Department of Meteorology and Atmospheric Science, Pennsylvania State University, University Park, PA, 16802, USA

Correspondence to: Ravi Kant Pathak (ravikant@chem.gu.se)

Reproducibility of soot

For high quality data, reproducibility of the flame in all experiments was ensured. The mass and number concentrations were measured four times during the entire measurement to guarantee that the same soot particles

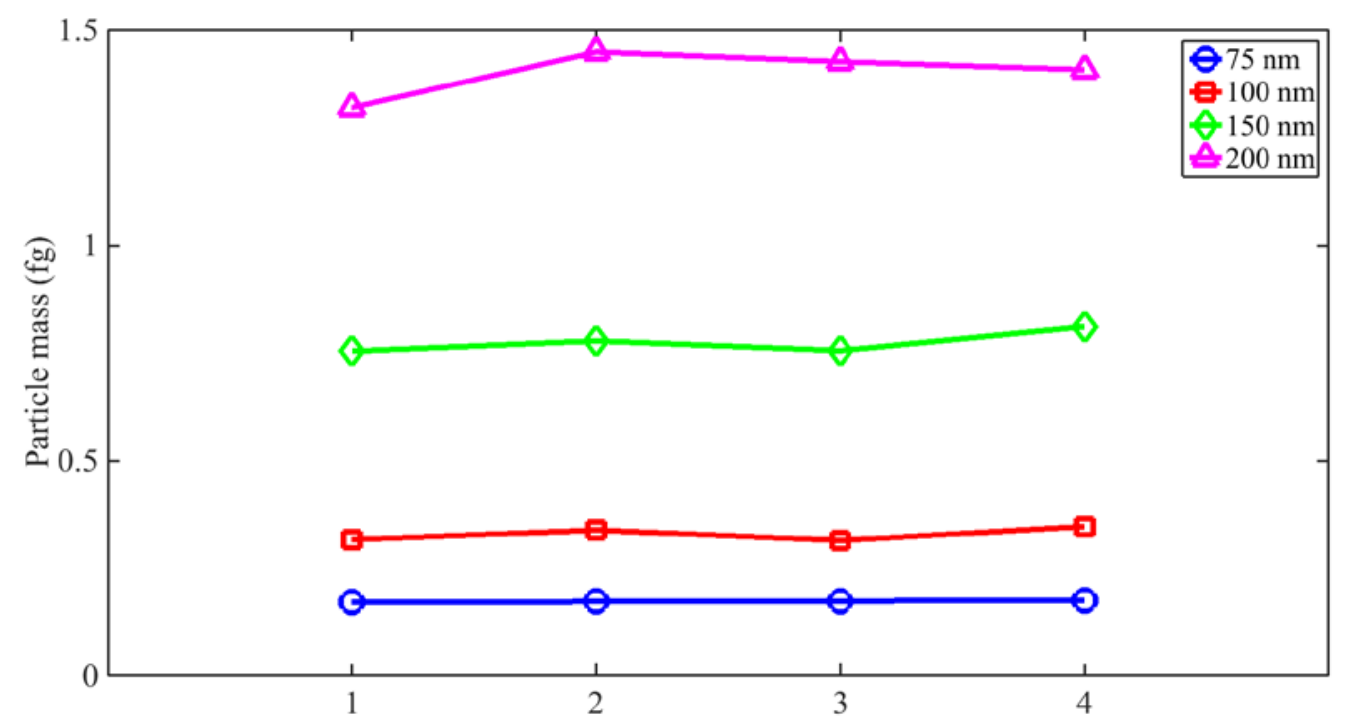

Figure S1. Particle mass of fresh soot with mobility size of 75, 100, 150, and $200 \mathrm{~nm}$ during the measurement. 


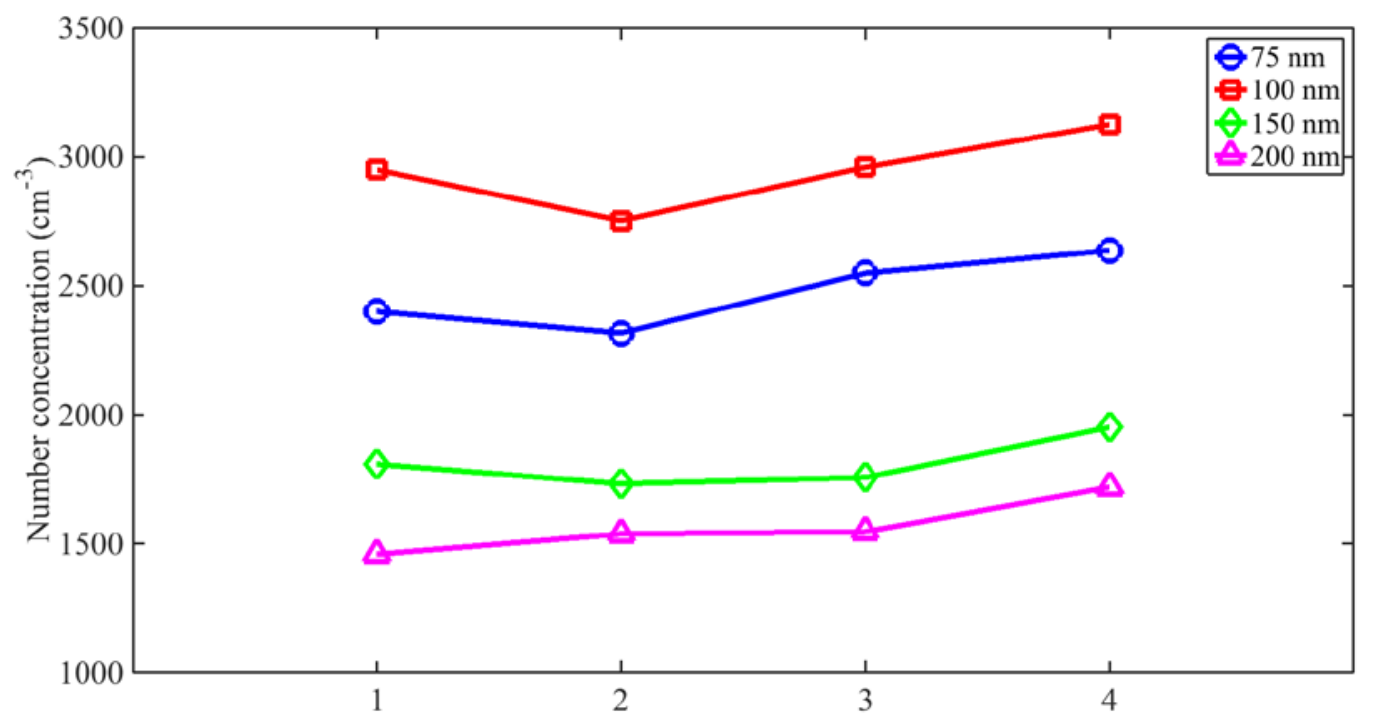

22

Figure S2. Number concentration of fresh soot with mobility size of 75, 100, 150, and $200 \mathrm{~nm}$ during the measurement.

\section{Correction of multiply charged particles}

The DMA-APM-CPC scans were fitted by normal distributions. The DMA-APM-CPC system can distinguish between singly and multiply charged (mainly doubly) spherical particles. However, for soot aggregates with effective densities that decrease with increasing mobility diameter, this distinction is possible in only some cases. The doubly charged particles may result in the overestimation of the particle mass and the effective density. The fraction of doubly charged particles may influence the mobility diameter of the soot aggregates if this diameter is smaller than the geometric mean diameter (GMD; in this study: $90 \mathrm{~nm}$ ) of the particle number size distribution. Therefore, a sensitivity test was conducted for the 75-nm soot, assuming a Boltzmann charge distribution. The overestimation of the mass of the $75-\mathrm{nm}$ soot was $<5 \%$, whereas the overestimation of the masses of the $100-\mathrm{nm}$, 150-nm, and 200-nm soot was negligible, which is consistent with the literature (Rissler et al., 2013).

\section{Experimental conditions}

Table S1 shows the experiments performed and results obtained under different coating conditions.

Table S1. Experiments performed and results obtained under different coating conditions. 


\begin{tabular}{|c|c|c|c|c|c|c|c|c|c|c|c|c|}
\hline Exp. & $\begin{array}{l}\text { Mobility } \\
\text { diameter } \\
\text { of fresh } \\
\text { soot } \\
(\mathrm{nm})\end{array}$ & $\begin{array}{l}\text { Sulfuric } \\
\text { acid bath } \\
\mathrm{T}\left({ }^{\circ} \mathrm{C}\right)\end{array}$ & $\begin{array}{l}\text { VOC } \\
\text { bath } \\
\mathrm{T} \\
\left({ }^{\circ} \mathrm{C}\right)\end{array}$ & $\begin{array}{l}\text { Soot } \\
\text { core } \\
\text { mass } \\
\text { (fg) }\end{array}$ & $\begin{array}{l}\text { Sulfuric } \\
\text { acid mass } \\
\text { (fg) }\end{array}$ & $\begin{array}{l}\text { SOA } \\
\text { mass } \\
(f g)\end{array}$ & $\begin{array}{c}\text { Limonene } \\
\text { concentration } \\
\left(\mu \mathrm{g} \cdot \mathrm{m}^{-3}\right)\end{array}$ & $\begin{array}{l}\text { Coating } \\
\text { thickness, } \\
\Delta r_{m e}(\mathrm{~nm})\end{array}$ & $\begin{array}{l}\text { Diameter } \\
\text { growth } \\
\text { factor, } \\
\text { Gfd }\end{array}$ & $\begin{array}{l}\text { Effective } \\
\text { density, } \\
\rho_{\text {eff }} \\
\left(\mathrm{g} \cdot \mathrm{cm}^{-3}\right)\end{array}$ & $\begin{array}{l}\text { Dynamic } \\
\text { shape } \\
\text { factor, } \chi\end{array}$ & $\begin{array}{l}\text { Void } \\
\text { space } \\
\text { fraction, } \\
F_{v s}\end{array}$ \\
\hline 1 & 75 & bypass & $\begin{array}{l}\text { UV } \\
\text { off }\end{array}$ & 0.17 & 0.00 & 0.00 & 0 & 0 & 1.00 & 0.80 & 1.62 & 0.58 \\
\hline 2 & 75 & bypass & 1 & 0.17 & 0.00 & 0.08 & 56 & 5.7 & 1.11 & 0.88 & 1.39 & 0.45 \\
\hline 3 & 75 & bypass & 5 & 0.17 & 0.00 & 0.11 & 73 & 7.4 & 1.12 & 0.99 & 1.27 & 0.37 \\
\hline 4 & 75 & 1 & $\begin{array}{l}\text { UV } \\
\text { off }\end{array}$ & 0.17 & 0.02 & 0.00 & 0 & 1.4 & 1.00 & 0.86 & 1.55 & 0.52 \\
\hline 5 & 75 & 1 & 1 & 0.17 & 0.02 & 0.13 & 56 & 8.9 & 1.14 & 1.02 & 1.26 & 0.35 \\
\hline 6 & 75 & 1 & 5 & 0.17 & 0.02 & 0.12 & 73 & 8.5 & 1.16 & 0.96 & 1.31 & 0.40 \\
\hline 7 & 75 & 1 & 15 & 0.17 & 0.02 & 0.63 & 138 & 24.8 & 1.58 & 0.96 & 1.19 & 0.29 \\
\hline 8 & 75 & 5 & $\begin{array}{l}\text { UV } \\
\text { off }\end{array}$ & 0.17 & 0.03 & 0.00 & 0 & 1.3 & 0.98 & 0.86 & 1.55 & 0.53 \\
\hline 9 & 75 & 5 & 1 & 0.17 & 0.03 & 0.15 & 56 & 9.8 & 1.13 & 0.98 & 1.27 & 0.33 \\
\hline 10 & 75 & 5 & 5 & 0.17 & 0.03 & 0.26 & 73 & 14.3 & 1.22 & 1.03 & 1.20 & 0.26 \\
\hline 11 & 75 & 5 & 15 & 0.17 & 0.03 & 0.91 & 138 & 30.6 & 1.59 & 1.05 & 1.11 & 0.13 \\
\hline 12 & 75 & 25 & $\begin{array}{l}\text { UV } \\
\text { off }\end{array}$ & 0.17 & 0.26 & 0.00 & 0 & 9.9 & 1.19 & 1.20 & 1.27 & 0.37 \\
\hline 13 & 75 & 25 & 1 & 0.17 & 0.26 & 0.61 & 56 & 27.6 & 1.64 & 1.09 & 1.15 & 0.25 \\
\hline 14 & 75 & 25 & 5 & 0.17 & 0.26 & 0.70 & 73 & 29.4 & 1.71 & 1.08 & 1.15 & 0.27 \\
\hline 15 & 75 & 25 & 15 & 0.17 & 0.26 & 2.37 & 138 & 52.1 & 2.11 & 1.11 & 1.07 & 0.05 \\
\hline 16 & 100 & bypass & $\begin{array}{l}\text { UV } \\
\text { off }\end{array}$ & 0.33 & 0.00 & 0.00 & 0 & 0 & 1.00 & 0.64 & 1.83 & 0.63 \\
\hline 17 & 100 & bypass & 1 & 0.33 & 0.00 & 0.15 & 56 & 4.9 & 1.07 & 0.85 & 1.55 & 0.56 \\
\hline 18 & 100 & bypass & 5 & 0.33 & 0.00 & 0.18 & 73 & 6.4 & 1.10 & 0.82 & 1.54 & 0.54 \\
\hline 19 & 100 & bypass & 15 & 0.33 & 0.00 & 0.35 & 138 & 12.1 & 1.18 & 0.90 & 1.37 & 0.45 \\
\hline 20 & 100 & 1 & $\begin{array}{l}\text { UV } \\
\text { off }\end{array}$ & 0.33 & 0.01 & 0.00 & 0 & 0.1 & 1.01 & 0.69 & 1.74 & 0.63 \\
\hline 21 & 100 & 1 & 1 & 0.33 & 0.01 & 0.19 & 56 & 8.2 & 1.10 & 0.86 & 1.39 & 0.47 \\
\hline 22 & 100 & 1 & 5 & 0.33 & 0.01 & 0.20 & 73 & 8.5 & 1.13 & 0.81 & 1.44 & 0.50 \\
\hline 23 & 100 & 1 & 15 & 0.33 & 0.01 & 0.39 & 138 & 14.2 & 1.19 & 0.97 & 1.24 & 0.39 \\
\hline 24 & 100 & 5 & $\begin{array}{l}\text { UV } \\
\text { off }\end{array}$ & 0.33 & 0.04 & 0.00 & 0 & 2.0 & 1.01 & 0.67 & 1.78 & 0.60 \\
\hline 25 & 100 & 5 & 1 & 0.33 & 0.04 & 0.20 & 56 & 10.0 & 1.10 & 0.85 & 1.40 & 0.45 \\
\hline 26 & 100 & 5 & 5 & 0.33 & 0.04 & 0.24 & 73 & 11.1 & 1.13 & 0.90 & 1.35 & 0.46 \\
\hline 27 & 100 & 5 & 15 & 0.33 & 0.04 & 0.52 & 138 & 18.7 & 1.23 & 0.94 & 1.25 & 0.32 \\
\hline 28 & 100 & 25 & $\begin{array}{l}\text { UV } \\
\text { off }\end{array}$ & 0.33 & 0.34 & 0.00 & 0 & 8.8 & 1.07 & 1.08 & 1.35 & 0.43 \\
\hline 29 & 100 & 25 & 1 & 0.33 & 0.34 & 0.27 & 56 & 16.2 & 1.23 & 1.12 & 1.22 & 0.39 \\
\hline 30 & 100 & 25 & 5 & 0.33 & 0.34 & 0.45 & 73 & 20.3 & 1.34 & 1.07 & 1.21 & 0.41 \\
\hline
\end{tabular}




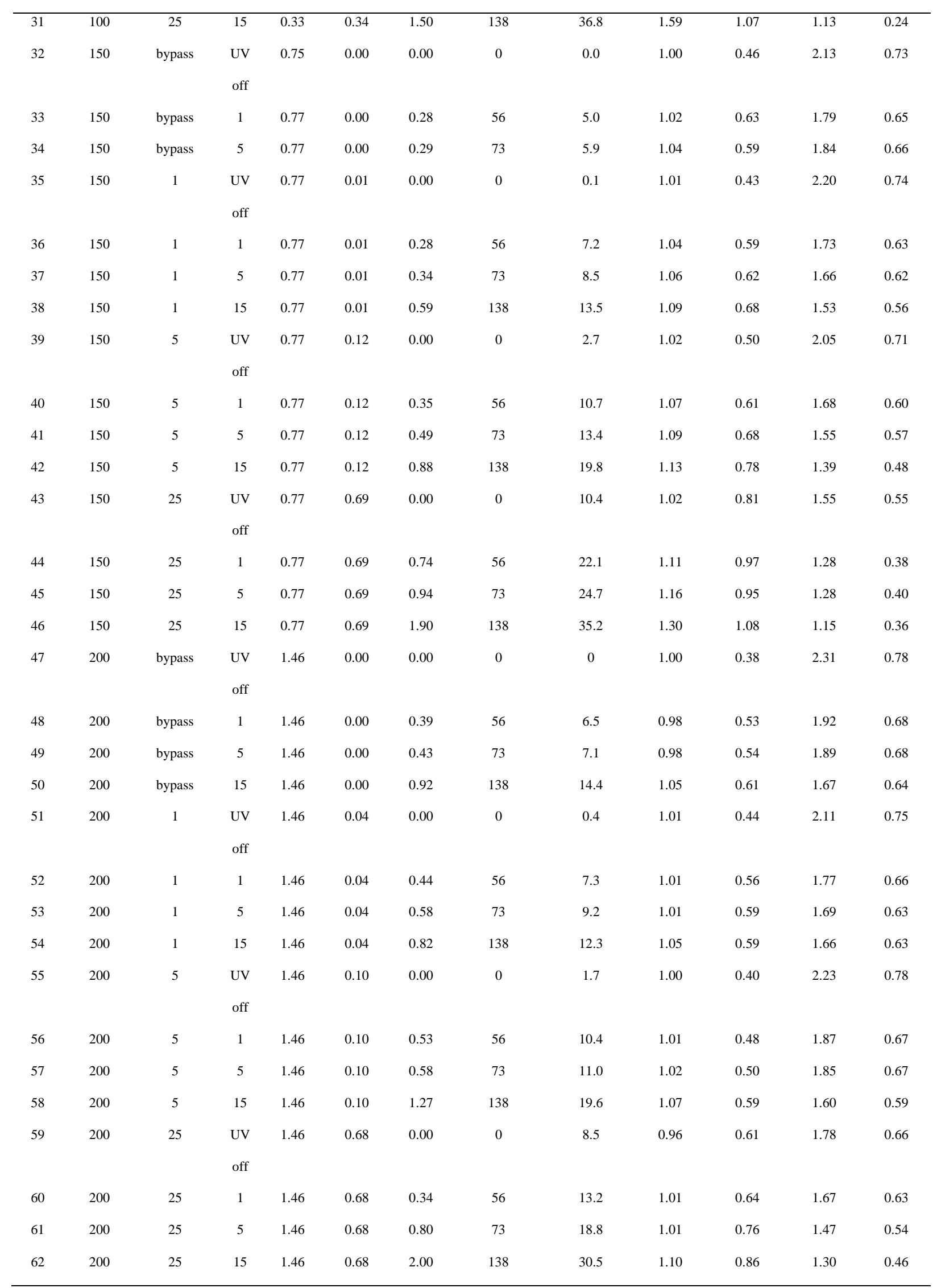




\begin{tabular}{|c|c|c|c|c|c|c|c|c|c|c|}
\hline Exp. & $\begin{array}{l}\text { Mobility } \\
\text { diameter of } \\
\text { fresh soot } \\
(\mathrm{nm})\end{array}$ & $\begin{array}{l}\text { Sulfuric } \\
\text { acid bath } \mathrm{T} \\
\left({ }^{\circ} \mathrm{C}\right)\end{array}$ & $\begin{array}{l}\text { VOC bath T } \\
\left({ }^{\circ} \mathrm{C}\right)\end{array}$ & $\begin{array}{l}\text { Void space } \\
\text { fraction, } \\
F_{v s}\end{array}$ & $\begin{array}{l}\text { Coating } \\
\text { thickness, } \\
\Delta r_{m e}(\mathrm{~nm})\end{array}$ & $\begin{array}{l}\Delta r_{m e} \\
\text { for } \\
\text { filling } \\
(\mathrm{nm})\end{array}$ & $\begin{array}{l}\Delta r_{m e} \text { for } \\
\text { growth (nm) }\end{array}$ & $\begin{array}{l}\text { Void } \\
\text { space } \\
\text { filled (\%) }\end{array}$ & $\begin{array}{l}\text { \% of } \\
\text { material } \\
\text { consumed } \\
\text { during } \\
\text { filling }\end{array}$ & $\begin{array}{l}\text { \% of } \\
\text { material } \\
\text { consumed } \\
\text { during } \\
\text { growth }\end{array}$ \\
\hline 1 & 75 & bypass & UV off & 0.58 & 0.0 & 0 & 0 & 0 & 0 & 0 \\
\hline 2 & 75 & bypass & 1 & 0.45 & 5.7 & 1.5 & 4.2 & 12 & 23 & 77 \\
\hline 3 & 75 & bypass & 5 & 0.37 & 7.4 & 3.0 & 4.4 & 26 & 35 & 65 \\
\hline 4 & 75 & 1 & UV off & 0.52 & 1.4 & 1.3 & 0.1 & 10 & 93 & 7 \\
\hline 5 & 75 & 1 & 1 & 0.35 & 8.9 & 3.6 & 5.3 & 31 & 34 & 66 \\
\hline 6 & 75 & 1 & 5 & 0.40 & 8.5 & 2.5 & 6.0 & 21 & 24 & 76 \\
\hline 7 & 75 & 1 & 15 & 0.29 & 24.8 & 3.3 & 21.5 & 28 & 7 & 93 \\
\hline 8 & 75 & 5 & UV off & 0.53 & 1.3 & 1.3 & 0 & 10 & 100 & 0 \\
\hline 9 & 75 & 5 & 1 & 0.33 & 9.8 & 5.0 & 4.8 & 46 & 44 & 56 \\
\hline 10 & 75 & 5 & 5 & 0.26 & 14.3 & 6.2 & 8.1 & 59 & 34 & 66 \\
\hline 11 & 75 & 5 & 15 & 0.13 & 30.6 & 8.4 & 22.2 & 86 & 15 & 85 \\
\hline 12 & 75 & 25 & UV off & 0.37 & 9.9 & 2.9 & 7.0 & 25 & 23 & 77 \\
\hline 13 & 75 & 25 & 1 & 0.25 & 27.6 & 3.7 & 23.9 & 32 & 7 & 93 \\
\hline 14 & 75 & 25 & 5 & 0.27 & 29.4 & 2.8 & 26.6 & 24 & 4 & 96 \\
\hline 15 & 75 & 25 & 15 & 0.05 & 52.1 & 9.3 & 42.8 & 97 & 6 & 94 \\
\hline 16 & 100 & bypass & UV off & 0.63 & 0.0 & 0 & 0 & 0 & 0 & 0 \\
\hline 17 & 100 & bypass & 1 & 0.56 & 4.9 & 1.2 & 3.7 & 6 & 22 & 78 \\
\hline 18 & 100 & bypass & 5 & 0.54 & 6.4 & 1.7 & 4.7 & 9 & 23 & 77 \\
\hline 19 & 100 & bypass & 15 & 0.45 & 12.1 & 3.1 & 9.0 & 17 & 20 & 80 \\
\hline 20 & 100 & 1 & UV off & 0.63 & 0.1 & 0.1 & 0 & 0 & 100 & 0 \\
\hline 21 & 100 & 1 & 1 & 0.47 & 8.2 & 3.1 & 5.1 & 17 & 33 & 67 \\
\hline 22 & 100 & 1 & 5 & 0.50 & 8.5 & 2.0 & 6.5 & 10 & 20 & 80 \\
\hline 23 & 100 & 1 & 15 & 0.39 & 14.2 & 4.6 & 9.6 & 26 & 25 & 75 \\
\hline 24 & 100 & 5 & UV off & 0.60 & 2.0 & 1.7 & 0.3 & 9 & 84 & 16 \\
\hline 25 & 100 & 5 & 1 & 0.45 & 10.0 & 4.9 & 5.1 & 28 & 43 & 57 \\
\hline 26 & 100 & 5 & 5 & 0.46 & 11.1 & 4.4 & 6.7 & 24 & 33 & 67 \\
\hline 27 & 100 & 5 & 15 & 0.32 & 18.7 & 7.3 & 11.4 & 44 & 29 & 71 \\
\hline 28 & 100 & 25 & UV off & 0.43 & 8.8 & 5.2 & 3.6 & 30 & 54 & 46 \\
\hline 29 & 100 & 25 & 1 & 0.39 & 16.2 & 4.6 & 11.6 & 26 & 21 & 79 \\
\hline 30 & 100 & 25 & 5 & 0.41 & 20.3 & 3.4 & 16.9 & 18 & 11 & 89 \\
\hline 31 & 100 & 25 & 15 & 0.24 & 36.8 & 7.3 & 29.5 & 44 & 10 & 90 \\
\hline 32 & 150 & bypass & UV off & 0.73 & 0.0 & 0 & 0 & 0 & 0 & 0 \\
\hline 33 & 150 & bypass & 1 & 0.65 & 5.0 & 3.6 & 1.4 & 9 & 70 & 30 \\
\hline 34 & 150 & bypass & 5 & 0.66 & 5.9 & 3.0 & 2.9 & 8 & 48 & 52 \\
\hline 35 & 150 & 1 & UV off & 0.74 & 0.1 & 0.1 & 0 & 0 & 100 & 0 \\
\hline 36 & 150 & 1 & 1 & 0.63 & 7.2 & 4.2 & 3.0 & 11 & 55 & 45 \\
\hline
\end{tabular}




\begin{tabular}{|c|c|c|c|c|c|c|c|c|c|c|}
\hline 37 & 150 & 1 & 5 & 0.62 & 8.5 & 4.3 & 4.2 & 11 & 47 & 53 \\
\hline 38 & 150 & 1 & 15 & 0.56 & 13.5 & 6.4 & 7.1 & 18 & 41 & 59 \\
\hline 39 & 150 & 5 & UV off & 0.71 & 2.7 & 1.1 & 1.6 & 3 & 39 & 61 \\
\hline 40 & 150 & 5 & 1 & 0.60 & 10.7 & 5.8 & 4.9 & 16 & 49 & 51 \\
\hline 41 & 150 & 5 & 5 & 0.57 & 13.4 & 6.9 & 6.5 & 19 & 45 & 55 \\
\hline 42 & 150 & 5 & 15 & 0.48 & 19.8 & 9.8 & 10.0 & 29 & 41 & 59 \\
\hline 43 & 150 & 25 & UV off & 0.55 & 10.4 & 8.7 & 1.7 & 25 & 81 & 19 \\
\hline 44 & 150 & 25 & 1 & 0.38 & 22.1 & 14.0 & 8.1 & 45 & 55 & 45 \\
\hline 45 & 150 & 25 & 5 & 0.40 & 24.7 & 12.6 & 12.1 & 39 & 41 & 59 \\
\hline 46 & 150 & 25 & 15 & 0.36 & 35.2 & 12.4 & 22.8 & 39 & 24 & 76 \\
\hline 47 & 200 & bypass & UV off & 0.78 & 0.0 & 0 & 0 & 0 & 0 & 0 \\
\hline 48 & 200 & bypass & 1 & 0.68 & 6.5 & 6.5 & 0 & 10 & 100 & 0 \\
\hline 49 & 200 & bypass & 5 & 0.68 & 7.1 & 7.1 & 0 & 12 & 100 & 0 \\
\hline 50 & 200 & bypass & 15 & 0.64 & 14.4 & 9.2 & 5.2 & 15 & 59 & 41 \\
\hline 51 & 200 & 1 & UV off & 0.75 & 0.4 & 0.4 & 0 & 1 & 100 & 0 \\
\hline 52 & 200 & 1 & 1 & 0.66 & 7.3 & 7.3 & 0 & 12 & 100 & 0 \\
\hline 53 & 200 & 1 & 5 & 0.63 & 9.2 & 8.4 & 0.8 & 14 & 90 & 10 \\
\hline 54 & 200 & 1 & 15 & 0.63 & 12.3 & 8.2 & 4.1 & 14 & 62 & 38 \\
\hline 55 & 200 & 5 & UV off & 0.78 & 1.7 & 1.4 & 0.3 & 2 & 82 & 18 \\
\hline 56 & 200 & 5 & 1 & 0.67 & 10.4 & 9.7 & 0.7 & 16 & 92 & 8 \\
\hline 57 & 200 & 5 & 5 & 0.67 & 11.0 & 9.4 & 1.6 & 16 & 83 & 17 \\
\hline 58 & 200 & 5 & 15 & 0.59 & 19.6 & 12.8 & 6.8 & 23 & 59 & 41 \\
\hline 59 & 200 & 25 & UV off & 0.66 & 8.5 & 8.5 & 0 & 14 & 100 & 0 \\
\hline 60 & 200 & 25 & 1 & 0.63 & 13.2 & 13.2 & 0 & 24 & 100 & 0 \\
\hline 61 & 200 & 25 & 5 & 0.54 & 18.8 & 18.1 & 0.7 & 35 & 95 & 5 \\
\hline 62 & 200 & 25 & 15 & 0.46 & 30.5 & 20.5 & 10.0 & 41 & 58 & 42 \\
\hline
\end{tabular}

42

\section{Particle growth in mass}

44 The particle mass was determined via DMA-APM. The particle mass growth factor, Gfm, is determined from:

$$
G f m=\frac{m_{p}}{m_{0}}
$$

45 where $m_{0}$ and $m_{p}$ represent the masses of particles before and after coating, respectively. 

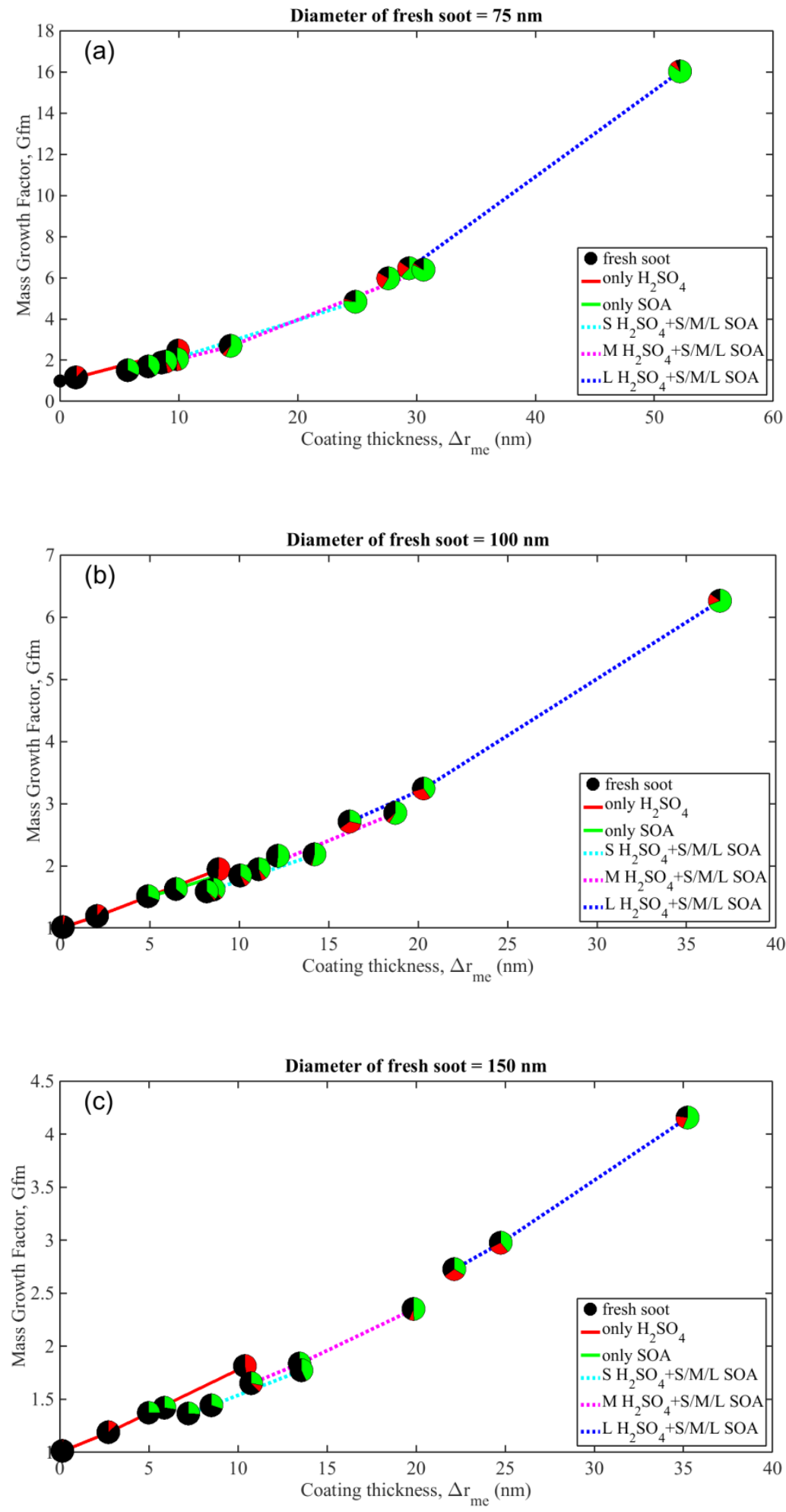


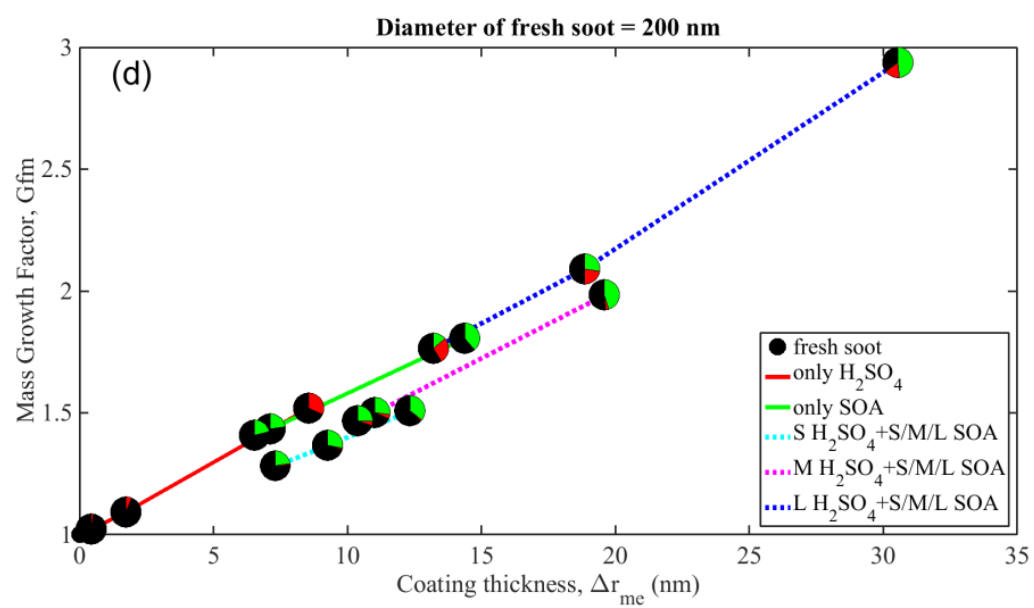

Figure S3 shows the growth of the particle mass $(G f m)$ for four sizes of fresh soot subjected to different coating conditions as a function of the mass equivalent coating thickness $\left(\Delta r_{m e}\right) . G f m$ increases significantly when the initial fresh soot size is small compared with the maximum size. Gfm increases to 16.00, 6.26, 4.15, and 2.94 for initial fresh-soot sizes of $75 \mathrm{~nm}, 100 \mathrm{~nm}, 150 \mathrm{~nm}$, and $200 \mathrm{~nm}$, respectively. This indicates that the core size of the initial soot can determine the evolution of $G f m$.

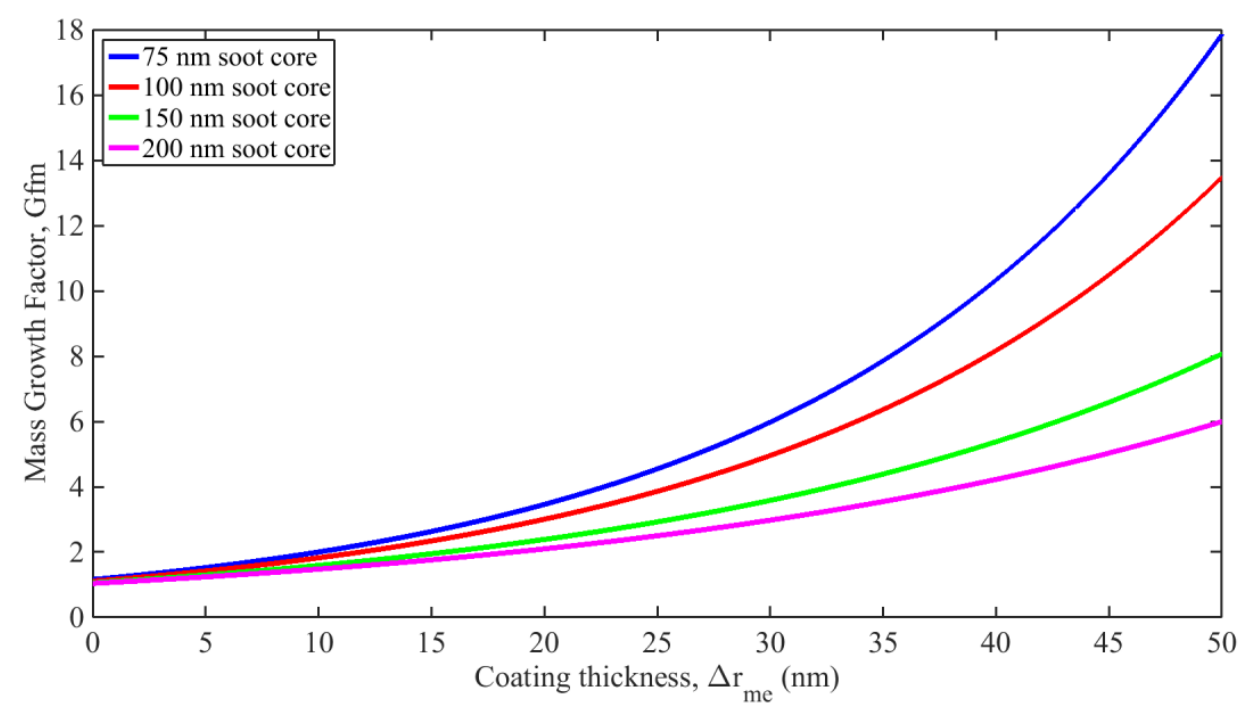


Using Eq. (3), Gfm was fitted as a function of $\Delta r_{m e}$.

$$
G f m=a \cdot \exp \left(b \cdot \Delta r_{m e}\right)
$$

63

Table S4. Morphology of fresh soot coated with various compounds, as illustrated in Fig. 5.

\begin{tabular}{|c|c|c|c|c|c|}
\hline Literature & Coating compound(s) & $D_{p, 0}(\mathrm{~nm})$ & $\begin{array}{l}D_{m e, 0} \\
(\mathrm{~nm})\end{array}$ & $D f m$ & $\begin{array}{l}d_{p p} \\
(\mathrm{~nm})\end{array}$ \\
\hline Pagels et al. (2009) & Sulfuric acid & 75 & 30.2 & 2.15 & 15 \\
\hline Xue et al. (2009) & $\begin{array}{l}\text { Glutaric acid (GA), } \\
\text { succinic acid (SA) }\end{array}$ & 80 & 32.9 & 2.20 & 16 \\
\hline Qiu et al. (2012) & $\begin{array}{l}\text { Toluene-OH oxidation } \\
\text { products }\end{array}$ & 100 & 61.9 & 2.17 & 21 \\
\hline Khalizov et al. (2013) & $\begin{array}{l}\text { Isoprene-OH oxidation } \\
\text { products }\end{array}$ & 100 & 61.9 & 2.20 & 19 \\
\hline Peng et al. (2016) & $\begin{array}{l}\text { SOA from ambient } \\
\text { precursors }\end{array}$ & 100 & 63.4 & 2.25 & 16 \\
\hline Guo et al. (2016) & $\begin{array}{l}\text { m-Xylene-OH } \\
\text { oxidation products }\end{array}$ & 100 & 61.4 & 2.17 & 45 \\
\hline $\begin{array}{l}\text { Ghazi and Olfert } \\
\text { (2013) }\end{array}$ & Dioctyl sebacate & 100 & 51.9 & 2.14 & 45 \\
\hline This study & $\begin{array}{l}\text { Sulfuric acid, acidity- } \\
\text { mediated limonene } \\
\text { SOA }\end{array}$ & 100 & 70.8 & 2.28 & 28 \\
\hline
\end{tabular}


67 Rissler, J., Messing, M. E., Malik, A. I., Nilsson, P. T., Nordin, E. Z., Bohgard, M., Sanati, M., and 68 Pagels, J. H.: Effective Density Characterization of Soot Agglomerates from Various Sources and 69 Comparison to Aggregation Theory, Aerosol Sci.Technol., 47, 792-805,

70 10.1080/02786826.2013.791381, 2013.

71 